(C) 2008 The Japan Society of Applied Physics

\title{
Organic Field-Effect Transistor Devices Based on Latent Pigments of Unsubstituted Diketopyrrolopyrrole or Quinacridone
}

\author{
Hiroyuki Yanagisawa, Jin Mizuguchi, Shinji Aramaki ${ }^{1}$, and Yoshimasa SaKai ${ }^{1}$ \\ Department of Applied Physics, Graduate School of Engineering, Yokohama National University, Yokohama 240-8501, Japan \\ ${ }^{1}$ Mitsubishi Chemical Group Science and Technology Research Center, Yokohama 240-8502, Japan
}

(Received December 30, 2007; accepted January 29, 2008; published online June 13, 2008)

Organic field-effect transistors (FETs) based on unsubstituted diketopyrrolopyrrole (DPP) or quinacridone (QA) have been fabricated using their solvent-soluble precursors called latent pigments ( $t$-BOC DPP and $t$-BOC QA) which can also be regenerated into their parent pigments by heating at about $200^{\circ} \mathrm{C}$. The use of latent pigments enables us to fabricate FETs by spin coating, offering a low-cost fabrication process rather than an expensive vacuum technology. The objective of the present investigation is to evaluate the performance of DPP- and QA-based FETs prepared using latent pigments. As a result, field effect mobilities of about $7.19 \times 10^{-6}$ and $8.23 \times 10^{-6} \mathrm{~cm}^{2} \mathrm{~V}^{-1} \mathrm{~s}^{-1}$ are obtained for FETs based on $t$-BOC DPP and QA, respectively. These values are almost equivalent to those of FETs prepared by the vacuum deposition of DPP and QA, namely $1.43 \times 10^{-5}$ and $1.08 \times 10^{-5} \mathrm{~cm}^{2} \mathrm{~V}^{-1} \mathrm{~s}^{-1}$, respectively. The present result leads us to conclude that latent pigment technology is an excellent low-cost process fabricating organic FETs. [DOI: 10.1143/JJAP.47.4728]

KEYWORDS: organic FET, latent pigment, diketopyrrolopyrrole, quinacridone, crystal structure

\section{Introduction}

Recently, a number of field-effect transistors (FETs) have been fabricated with thin films of organic materials. ${ }^{1,2)}$ The field-effect mobility of organic FETs is still much lower than that of inorganic FETs. However, organic FETs have certain advantages for low-cost and large-area device fabrication carried out at room temperature. Their target is therefore toward flexible display and IC tag applications, and solution processibility is one of the key issues for achieving low-cost FETs by spin coating.

We previously reported that a metal-free porphyrin, the so-called benzoporphyrin (BP), exhibits an excellent FET characteristic. $^{3)}$ Our FET system is characterized by the use of a solvent-soluble BP precursor and its thermal transformation into BP directly on the substrate at about $200^{\circ} \mathrm{C}$. Quite recently, we have also reported that copper-integrated BP (CuBP) shows an even better FET performance, as characterized by its high mobility of about $1.3 \mathrm{~cm}^{2} \mathrm{~V}^{-1} \mathrm{~s}^{-1}$.

In this study, we will present another type of solventsoluble precursor called the latent pigment ${ }^{5-7)}$ for fabricating organic FETs through the solution process. We deal here with two kinds of latent pigment based on diketopyrrolopyrrole [DPP; Fig. 1(a)] and quinacridone [QA; Fig. 1(b)] that are typical of hydrogen-bonded pigments: $t$-BOC DPP and $t$-BOC QA. DPP and QA are industrially important red colorants that belong to a class of hydrogen-bonded pigments. ${ }^{8)}$ In the solid state, there are chains of $\mathrm{NH} \cdots \mathrm{O}$ intermolecular hydrogen bonds between the $\mathrm{NH}$ group of one molecule and the $\mathrm{O}$ atom of the neighboring molecule, as shown in Figs. 2(a) ${ }^{9)}$ and 2(b). ${ }^{10)} \mathrm{NH} \cdots \mathrm{O}$ hydrogen bonds have two important functions. ${ }^{11)}$ One is to align the transition dipole in a head-to-tail fashion to displace the absorption band toward longer wavelengths in going from the solution state to the solid state. The other is to bridge small molecules of pigments together to impart a polymerlike stability.

The latent pigment has been developed by Zambounis of CIBA Specialty Chemicals. ${ }^{5,6)}$ A soluble precursor is prepared by replacing the $\mathrm{H}$ atom of the $\mathrm{NH}$ group with a $t$-butoxycarbonyl ( $t$-BOC) group. The insoluble parent (a)

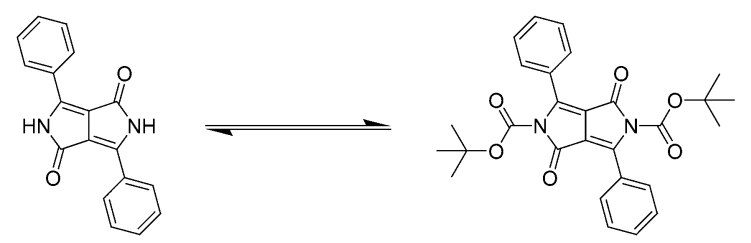

(b)

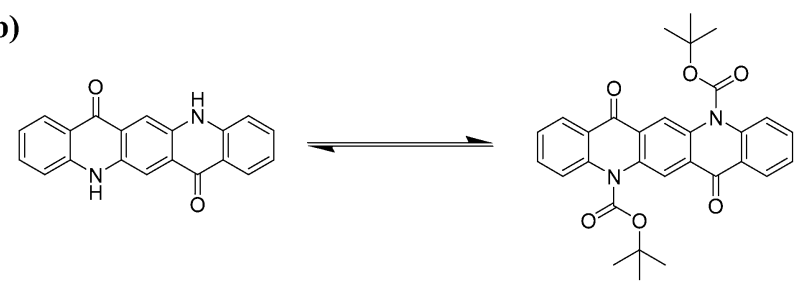

Fig. 1. Molecular structures: (a) DPP and t-BOC DPP and (b) QA and $t$-BOC QA.

pigment can then be regenerated by the thermal treatment of the precursor at about $180-200^{\circ} \mathrm{C}$. The regeneration process of $t$-BOC $\mathrm{DPP}^{12)}$ and $\mathrm{QA}^{13)}$ as well as their crystal structures $^{14-16)}$ have extensively been studied.

Our goal is to fabricate organic FETs using latent pigments of DPP or QA, and compare their performance with that of vacuum-deposited FETs of commercial DPP and QA.

\section{Experimental Procedure}

\subsection{Preparation of latent pigments}

$t$-BOC DPP and QA were prepared using DPP or QA (both of which are from CIBA Specialty Chemicals) as previously reported. ${ }^{5,6)}$ Both $t$-BOC products were yellowish powders.

\subsection{Fabrication of FETs}

Two kinds of FET were fabricated: spin-coated FET with latent pigments and vacuum-evaporated FET with commercial pigments.

The FET structure is shown in Fig. 3. A $\mathrm{SiO}_{2}$ layer of about $300 \mathrm{~nm}$ was thermally grown on a heavily doped ntype $\mathrm{Si}$-substrate that serves as the gate electrode. On top of 
(a)

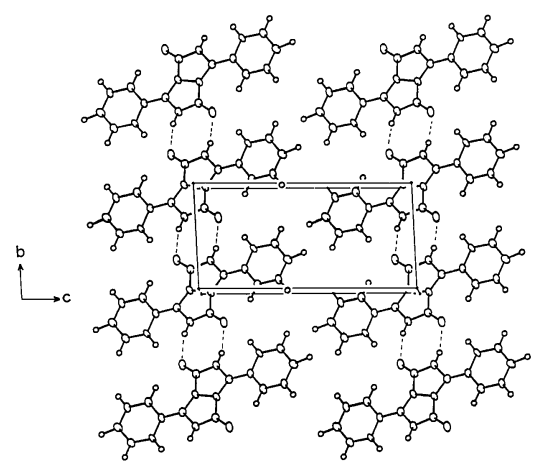

(b)

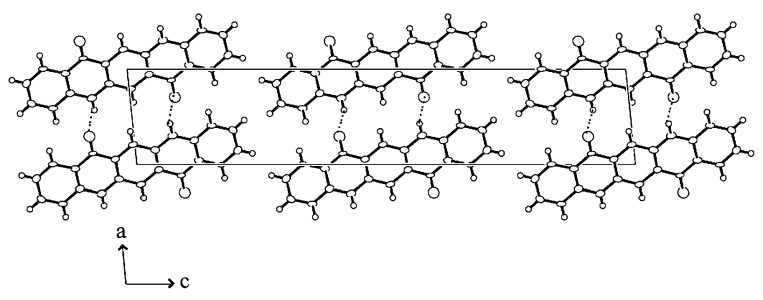

Fig. 2. Molecular arrangements: (a) DPP and (b) $\beta$-QA.

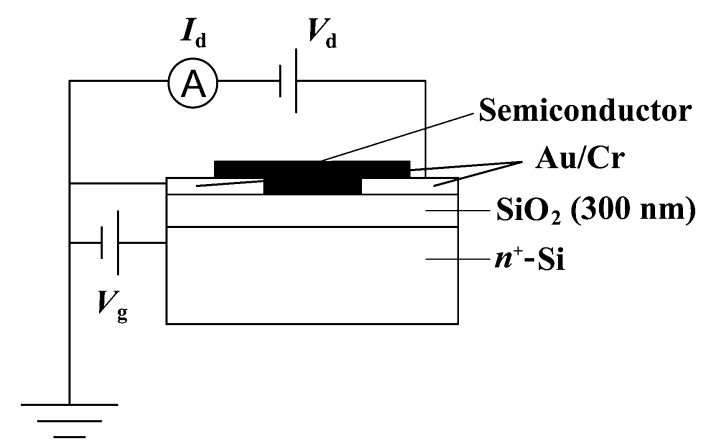

Fig. 3. Structure of FET.

$\mathrm{SiO}_{2}$, the source and drain electrodes made of $\mathrm{Au}(1000 \AA)$ / $\mathrm{Cr}(50 \AA)$ were prepared by photolithographic technology. The channel length and channel width were 10 and $500 \mu \mathrm{m}$, respectively.

No special surface modification for the substrate was made before spin coating. Spin coating was carried out on the channel of the FET substrates using chloroform solution of $t$-BOC DPP $(8.7 \mathrm{wt} \%)$ or $t$-BOC QA $(8.7 \mathrm{wt} \%)$ at a rate of $1000 \mathrm{rpm}$ for $30 \mathrm{~s}$. Then, the film was converted into DPP or QA by heating at $220^{\circ} \mathrm{C}$ for $15 \mathrm{~min}$. The phase of regenerated DPP was in fairly good agreement with that of single crystals. ${ }^{9,12)}$ On the other hand, regenerated QA was always of the $\beta$-form. ${ }^{10,13)}$

Vacuum-deposited films were prepared as follows: Commercial DPP and $\beta$-QA were purified three times by sublimation under vacuum, and then evaporated onto the channel of the FET substrates. The thickness of evaporated films was about $1000 \AA$.

\subsection{Equipment}

The current-voltage $(I-V)$ characteristics of the FETs were measured under vacuum with an Agilent $4155 \mathrm{C}$ semiconductor parameter analyzer. Scanning electron mi-

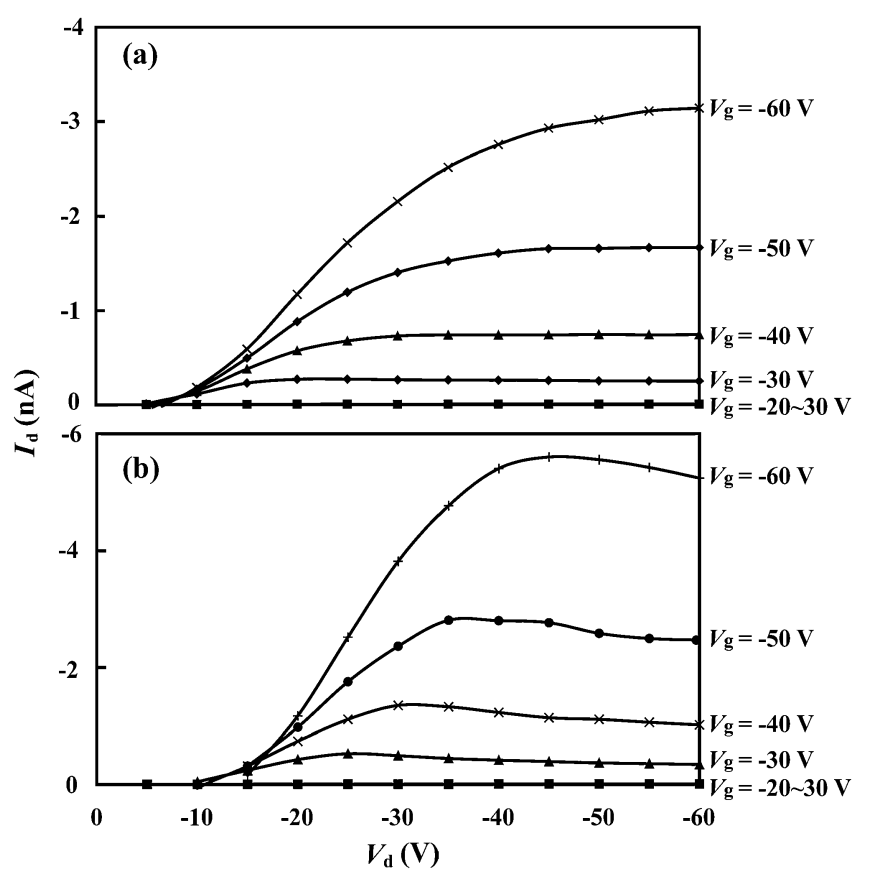

Fig. 4. $I-V$ characteristics: (a) spin-coated FET based on DPP and (b) vacuum-deposited FET based on commercial DPP.

croscopy (SEM) images were taken using a VE-8800 REAL 3D system from KEYENCE. Powder X-ray diffraction diagrams were measured on a Rigaku diffractometer (model: Rapid F). X-ray diffraction diagrams of the thin films were measured on a Rigaku RINT2000.

\section{Results and Discussion}

\subsection{FET characteristics}

Field-effect mobility $(\mu)$ and threshold voltage $\left(V_{\mathrm{t}}\right)$ were deduced from the $I_{\mathrm{sat}}{ }^{1 / 2}-V_{\mathrm{g}}$ plot on the basis of the equation $I_{\text {sat }}=\left[\left(C_{\mathrm{i}} W\right) /(2 L)\right] \mu\left(V_{\mathrm{g}}-V_{\mathrm{t}}\right)^{2}$, where $I_{\mathrm{sat}}, C_{\mathrm{i}}, W$, and $L$ stand for the saturation current, the capacitance of the insulator layer per unit area, the channel width, and the channel length, respectively. ${ }^{3)}$ The on/off ratio is defined as the ratio of drain current at $V_{\mathrm{g}}=-50 \mathrm{~V}$ to that at $V_{\mathrm{g}}=$ $30 \mathrm{~V}$; drain voltage is kept constant at $V_{\mathrm{d}}=-30 \mathrm{~V}$.

Figures 4(a) and 4(b) show the drain current-drain voltage $\left(I_{\mathrm{d}}-V_{\mathrm{d}}\right)$ plot measured at room temperature under vacuum for the spin-coated and vacuum-deposited FETs based on DPP, respectively. The curves are typical FET characteristics and show a linear region at low $V_{\mathrm{d}}$ and a saturation region at high $V_{\mathrm{d}}$. The negative sign of the drain and gate voltages indicates a hole transport of DPP. The field effect mobilities are $7.19 \times$ $10^{-6}$ and $1.43 \times 10^{-5} \mathrm{~cm}^{2} \mathrm{~V}^{-1} \mathrm{~s}^{-1}$, and the on/off ratios are 105 and 326 for spin-coated and vacuum-deposited FETs, respectively. It is remarkable to note that the mobility and on/off ratio of the spin-coated FETs are as large as those of the vacuum-deposited FETs.

Likewise, Figs. 5(a) and 5(b) show the $I_{\mathrm{d}}-V_{\mathrm{d}}$ plot for the spin-coated and vacuum-deposited FETs based on QA, respectively. The field-effect mobilities are $8.23 \times 10^{-6}$ and $1.08 \times 10^{-5} \mathrm{~cm}^{2} \mathrm{~V}^{-1} \mathrm{~s}^{-1}$, and the on/off ratios are 39 and 45 , respectively. Here, the hole transport is again confirmed. In addition, no significant difference in FET performance is observed between the spin-coated and vacuum-deposited FETs. 


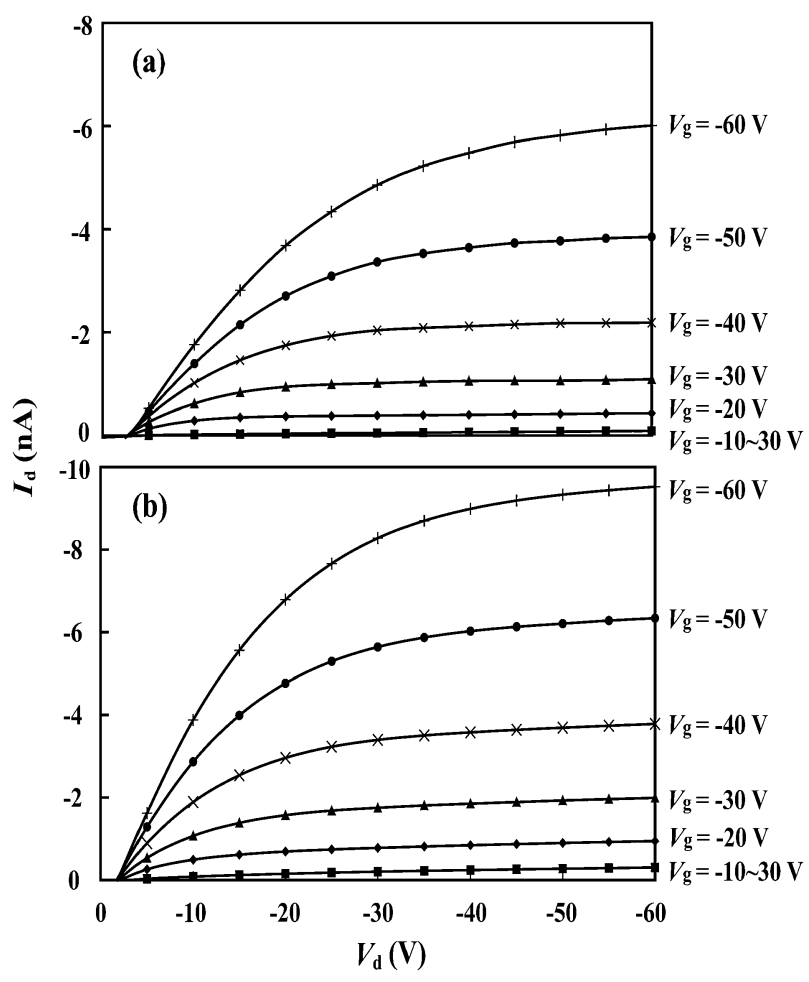

Fig. 5. I-V characteristics: (a) spin-coated FET based on QA and (b) vacuum-deposited FET based on $\beta$-QA.

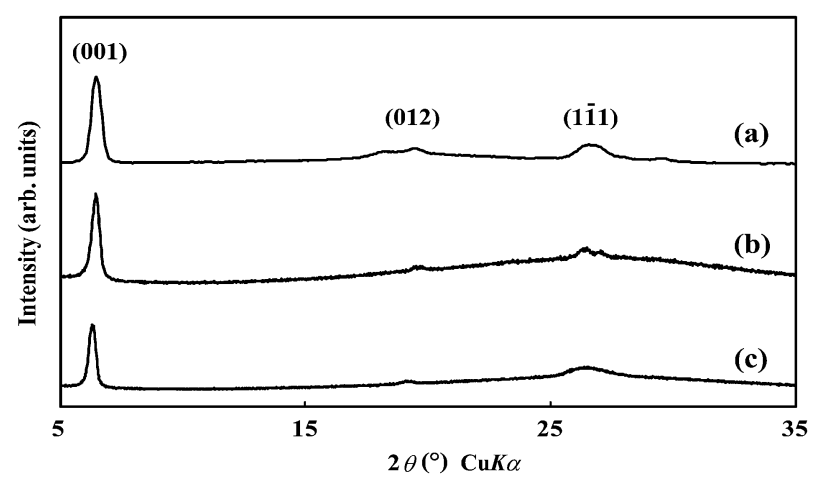

Fig. 6. X-ray diffraction diagrams: (a) powdered DPP, (b) spin-coated/ regenerated film of DPP on glass, and (c) evaporated DPP on glass.

\subsection{Crystalline state of DPP and QA films}

Figure 6 shows the X-ray diffraction diagrams of powdered DPP, spin-coated/regenerated film and vacuumdeposited film on glass substrate. The diffraction peaks are assigned on the basis of the crystallographic data of single crystals. ${ }^{14,15)}$ The diffraction peak at about $2 \theta=27^{\circ}[(1 \overline{1} 1)]$ correspond to the diffraction along the stacking axis of the molecules. In all the samples, the diffraction peaks of (001), (012), and (1 $\overline{1} 1)$ appear in common, and no significance difference is observed between the three diagrams. Furthermore, the molecules are not oriented in a particular direction with respect to the substrate, but are oriented in all directions as in the case of the powders [diagram (a)].

However, the situation is different for $\beta$-QA. Figure 7 shows the X-ray diffraction diagrams of the powdered $\beta$-QA, spin-coated/regenerated film and vacuum-deposited film on a glass substrate. The diffraction peaks are assigned on the

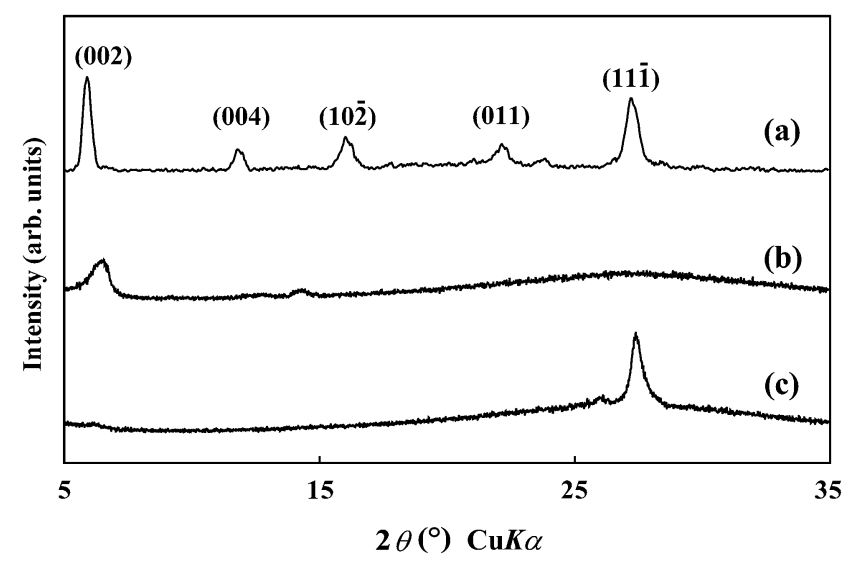

Fig. 7. X-ray diffraction diagrams: (a) powdered QA, (b) spin-coated/ regenerated film of QA on glass, and (c) evaporated $\beta$-QA on glass.

basis of the crystallographic data of a single crystal. ${ }^{16)}$ In the spin-coated/regenerated film, one small diffraction appears at $2 \theta=7^{\circ}$, which corresponds to the (002) plane, but no diffraction at about $27^{\circ}$. The indicates that the molecules are arranged along the $c$-axis perpendicular to the substrate [see Fig. 2(b)] and that the molecules are not ordered along the stacking $b$-axis. On the other hand, in vacuum-deposited films, only one diffraction peak at about $27^{\circ}[(11 \overline{1})]$, which corresponds to the diffraction along the stacking axis, was observed. This means that the molecules are stacked along the stacking $b$-axis parallel to the substrate. However, it is important to note that the present molecular orientation is not reflected in the FET performance of these two samples. That is, no correlation between the crystalline state and the FET characteristics is available at present in FETs based on QA.

The molecular orientation on a substrate plays an important role in FET performance. Therefore, the molecules should ideally be stacked parallel to the substrate, i.e., the direction of drain current. However, no clear correlation is obtained in the present experiment between molecular orientation and FET performance within our present experiment, particularly in QA. However, the surface of FET as observed by SEM shown below provides us with information on the difference in surface morphology between spincoated/regenerated and evaporated films.

\subsection{Surface morphology of FET as observed from SEM images}

Figures 8(a) and 8(b) show SEM images of FET for the spin-coated/regenerated and evaporated films of DPP, respectively. The surface of the regenerated $t$-BOC DPP is rough and uneven, characterized by a number of small needles. It is quite difficult to see the source, channel, and drain owing to the needles. This indicates that the molecules after regeneration were rather mobile and easily rearranged on the substrate in the crystallization process. On the other hand, the surface of the vacuum-deposited film is homogeneous and smooth, as is always the case for evaporated films of organic pigments. Also note that the surface morphology is not so important for the FET characteristic, but the bottom layer directly on the substrate plays a decisive role for the channel. 
(a)

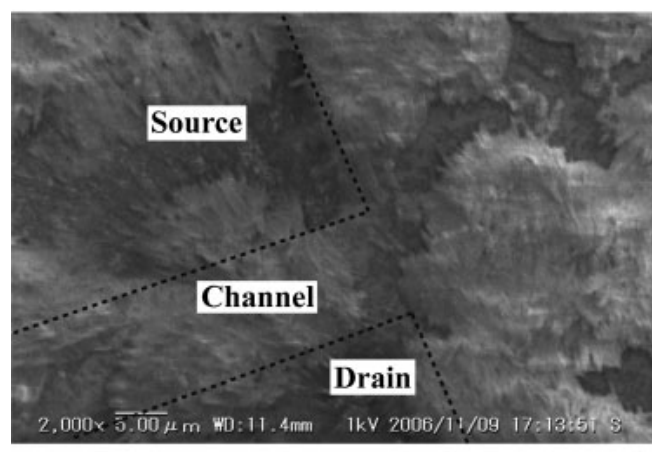

(b)

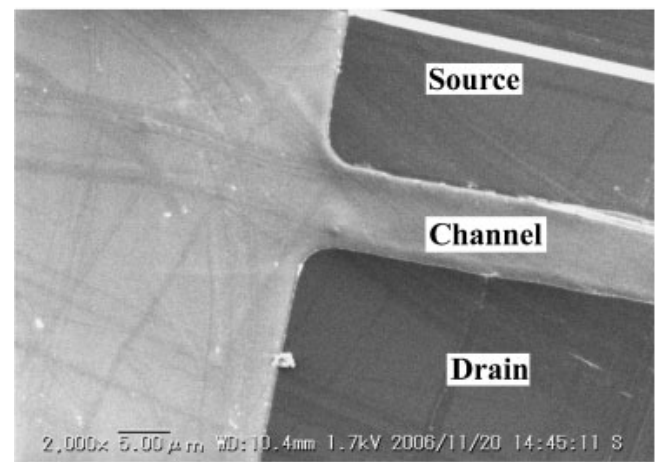

Fig. 8. SEM images: (a) spin-coated/regenerated film of DPP on glass and (b) evaporated DPP on glass.

Figures 9(a) and 9(b) show SEM images of FET for the spin-coated/regenerated and evaporated films of QA, respectively. The surface of the spin-coated/regenerated film is significantly more even than that of regenerated DPP. However, we see a number of voids that are indicative of the trace of gases (i.e., $\mathrm{CO}_{2}$ ) evolved during the regeneration process (see Fig. 1). The source, channel and drain can clearly be recognized even in the spin-coated/regenerated layer.

\section{Conclusions}

DPP- and QA-based FETs have been investigated on the basis of the latent pigment technology with particular attention to the low-cost fabrication process. The FET devices fabricated by $t$-BOC DPP and $t$-BOC QA are found to exhibit nearly equivalent FET characteristics to vacuumdeposited FET prepared by evaporating commercial DPP and QA. This suggests that $t$-BOC latent-pigment technology provides us with a new low-cost fabrication process for FETs. (a)

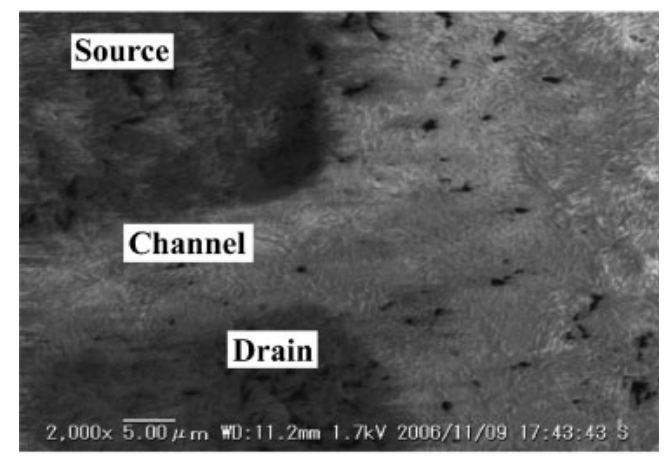

(b)

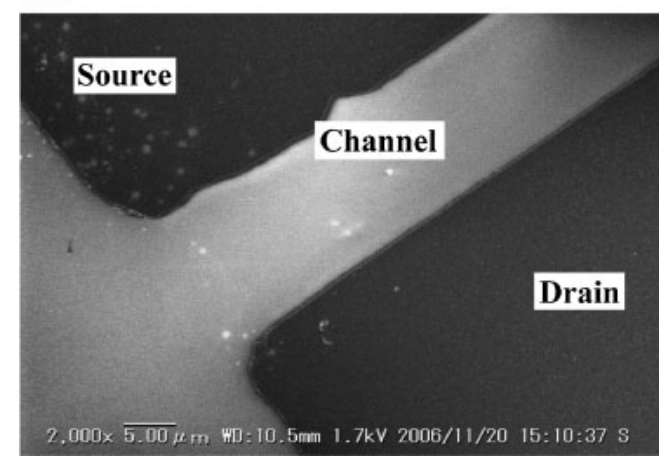

Fig. 9. SEM images: (a) spin-coated/regenerated film of QA on glass and (b) evaporated $\beta$-QA on glass.

1) C. C. Dimitrakopoulos, A. R. Brown, and A. Pomp: J. Appl. Phys. 80 (1996) 2501.

2) Z. Bao, A. J. Lovinger, and J. Brown: J. Am. Chem. Soc. 120 (1998) 207.

3) S. Aramaki, Y. Sakai, and N. Ono: Appl. Phys. Lett. 84 (2004) 2085.

4) S. Aramaki, A. Ohno, Y. Sakai, and R. Yoshiyama: Japan Patent 2006165533 A (2006).

5) J. Zambounis, Z. Hao, and A. Iqbal: Nature 388 (1997) 131.

6) J. Zambounis, Z. Hao, and A. Iqbal: US Patent 5484943 (1996).

7) J. Mizuguchi: Nihon Gazo Gakkaishi 44 (2005) 347 [in Japanese].

8) W. Herbst and K. Hunger: Industrial Organic Pigments (VCH, Weinheim, 1993) p. 550.

9) J. Mizuguchi, A. Grubenmann, G. Wooden, and G. Rihs: Acta Crystallogr., Sect. B 48 (1992) 696.

10) N. Nishimura, T. Senju, and Mizuguchi: Acta Crystallogr., Sect. E 62 (2006) 04683.

11) J. Mizuguchi: J. Phys. Chem. A 104 (2000) 1817.

12) J. Mizuguchi: J. Imaging Sci. Technol. 49 (2005) 35.

13) Y. Imura, Y. Yamashita, T. Senju, and J. Mizuguchi: Nihon Gazo Gakkaishi 44 (2005) 138.

14) J. Mizuguchi: Z. Kristallogr.-New Cryst. Struct. 218 (2003) 134.

15) J. Mizuguchi: Acta Crystallogr., Sect. E 59 (2003) o469.

16) J. Mizuguchi: Acta Crystallogr., Sect. E 59 (2003) o474. 